\title{
Enfisema Lobar Congénito (E.L.C.)
}

Dres. Jorge Rodríguez Ormazábal,* J. Peñailillo.* J. Zúñiga P.. ** G. Girardi, ${ }^{* *}$ J. Thūmber. ${ }^{* *} \mathrm{~S}$. Benveniste D. .****

T. H. Julia Cavada P.****

\begin{abstract}
Congenilal Lubar Emphisema is unt ul the causes of respiratory distress during infancy. We present three patients. sludied at the Dr. E. Cionzalez Curtes Hoxpitsl, during the periud comprebended bet ween 1973 and 1978.

All of them were intants under 6 munhs ot ase, with an asymplomatic period between birth ditd ibe onsel al symptoms: these consioted essentialy in a respiralory distress sindrone at progressive severity: chest $x$ rays simwed hipertranslucery with an thomogenemus trabecular sirue ture correspunaling in pulnunary parenchinn.

The three patients were operaled presenting a sutjisfaclury post-surgery out comte.

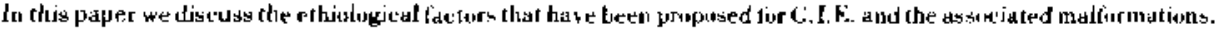

Afección cuya característica anatomopatológica es. triba en una sobredistensión difusa de los alvéulos pulmonares, de localización lobar.

Este cuadro fue descrito por Peller y Philips 1922 y la literatura tiende a agrupar bajo este concepto la terminología anterior de "Enfisema Unilobar Obstructivo". "Enfisema Lobar a Tensión de la Infancia", "Enfisema hipertrófico localizado de la Infancia".

\section{MATERIAL Y METODO}

Se presentan tres casos de E.L.C., controlados por e] Servicio de Broncopulmonares del Hospital Exequiel González Cortés en el periodo 1973-1978 y se analizan con respecto a su Cuadro Clinico, hallazgos radiológicos, operatorios y anatomía patológica.

\section{CASO 1:}

Eutrófico que consulta a $\operatorname{los} 21 / 2$ meses de edad por cuadro brusco de dificultad respiratoria, cianosis central, fiebre moderada. EF: Hiperinsuflación torácica, espiración prolongada, tiraje, crépitos basales bilaterales.

Rgx Tórax: (a.p.) (Rgk 1) Marcada hiperinsuflación del

\footnotetext{
* Médjuco becado Hospital E. Gonzàez Cortés.

** Servicio Brancopulmunares, Hospital E. Goncalez Cortés.

***servicio Radiulogía, Hospital E. González Curtés.

**** jervicio Anatomía Patológica, Hospilal E. Gunzález Curtés.
}

lóbulo superior izquierdo que desplaza el mediastimo hacia la derecha, comprimiendo al mismo tiempo el resto del pulmón izquierdo. Sombras de condensación perihiliar y paravertebral basal derecha. Diafragmas bajos aplanados.

Se trata con PNC-Kanamjcina, con buena respuesta desde el punto de vista de su Bronconemmonía. A los 34 días de su hospitalización, nueva reagudización de su cuadro pulmonar, que requiere cura antibiótica por 7 días.

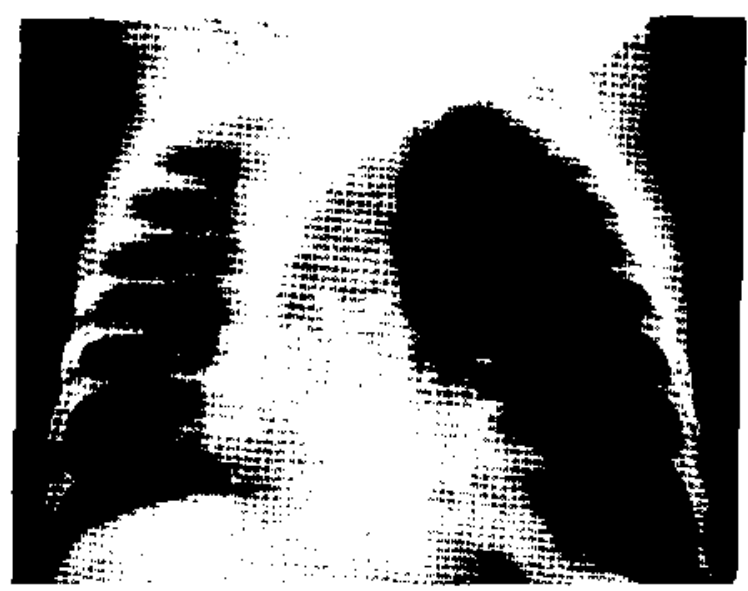

Tränsito esófago, estómago, duodeno = normal.

Se practica broncografia ( $\left.R_{\mathrm{gx}} 2\right)$, que concluye:

Bronquios del lóbulo inferior de calibre normal. Del lóbulo superior se observa el bronquio principal sólo hasta 2 a 3 centímetros distante de su fuente donde termina su lumen abruptamente. 


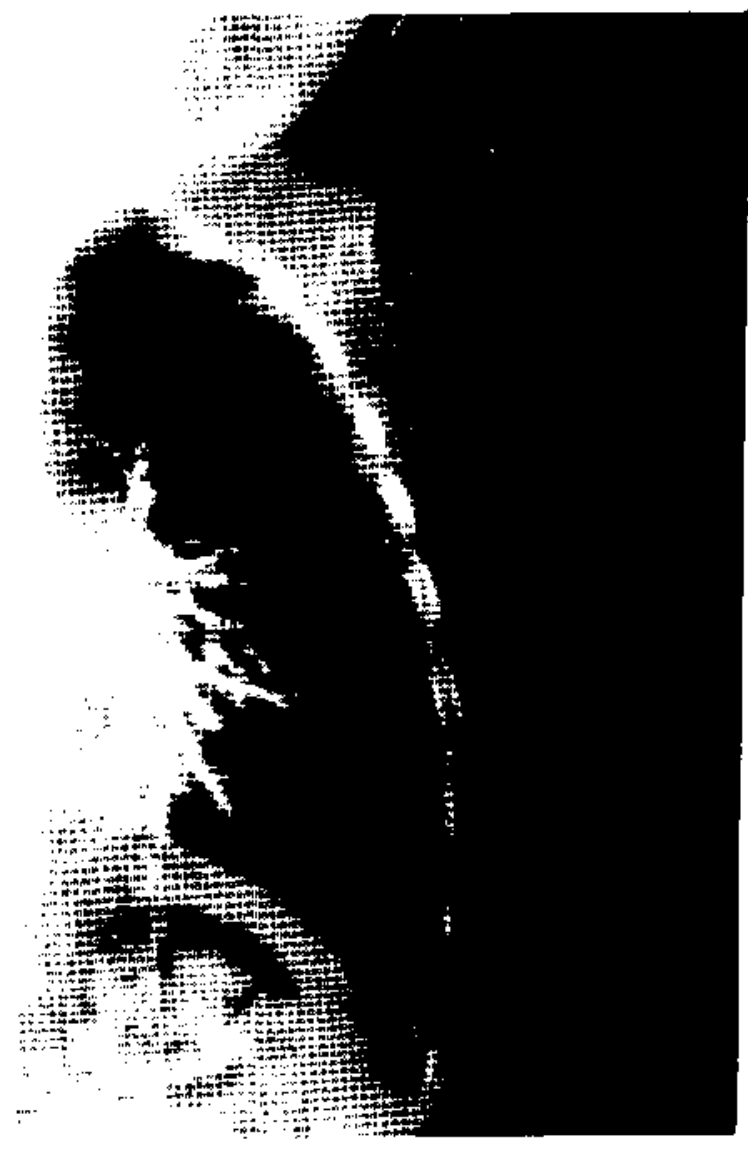

Sólo tres bronquios que nacen del bronquio principal y que se contrastan en forma irregular son visibles en el área enfisematosa. Desplazamiento de la tráquea hacia la derecha y moderado desplazamiento de los bronquios de la lingula hacia caudal.

Se plantea el diagnóstico de E,L.C., y se interviene quirúrgicamente, practicándose lobectomía superiơr izquierda. Durante la intervención se comprueba que aproximadamente el $80 \%$ del lóbulo se encuentra enfisematoso y que además del bronquio fuente, en lóbulo superior izquierdo existe un bronquio accesorio más pequeño de consistencia blanda que airea la zona de enfigema.

La biopsia concluye: presencia de alvéolos sobrediatendidos, los que coexisten con procesos de: bronconeumonía focal, ateleclasia y hemorragis pulmonares.

Se da de alta en buenas condiciones.

El control 3 a 11 meses después de la operación mựatra buena reexpansión del resto del pulmón izquierdo y adherencias pleurales especialmente mediastinales.

\section{CASO 2:}

Lactante de 5 l/2 meses de edad, sin antecedentes mórbidos previos, que ingresa con los diagnóstjcos de DCPI, probable corqueluche.

Dos dias anter de su ingreso presenta: accesos de tos quintosa, quejido, acompañado de cianosis.
Al examen físico: subfebril, polipneico, retracción inter y subcostal, roncus, sibilantes y estertores bronquiales diseminados.

Rgx Tórax: (Rgx 3 y 4) Marcada hjperinsuflación del lóbulo superior izquierdo con compresión de] resto del pulmón que se encuentra en la base del hemitórax. Herniación del lóbulo superior a través del mediastino hacia la cavidad torácica derecha con desplazamiento de los órganos mediastínicos en el misma sentido. Diafragmas algn aplanados. Aumento de los espacios intercostales izquierdos.
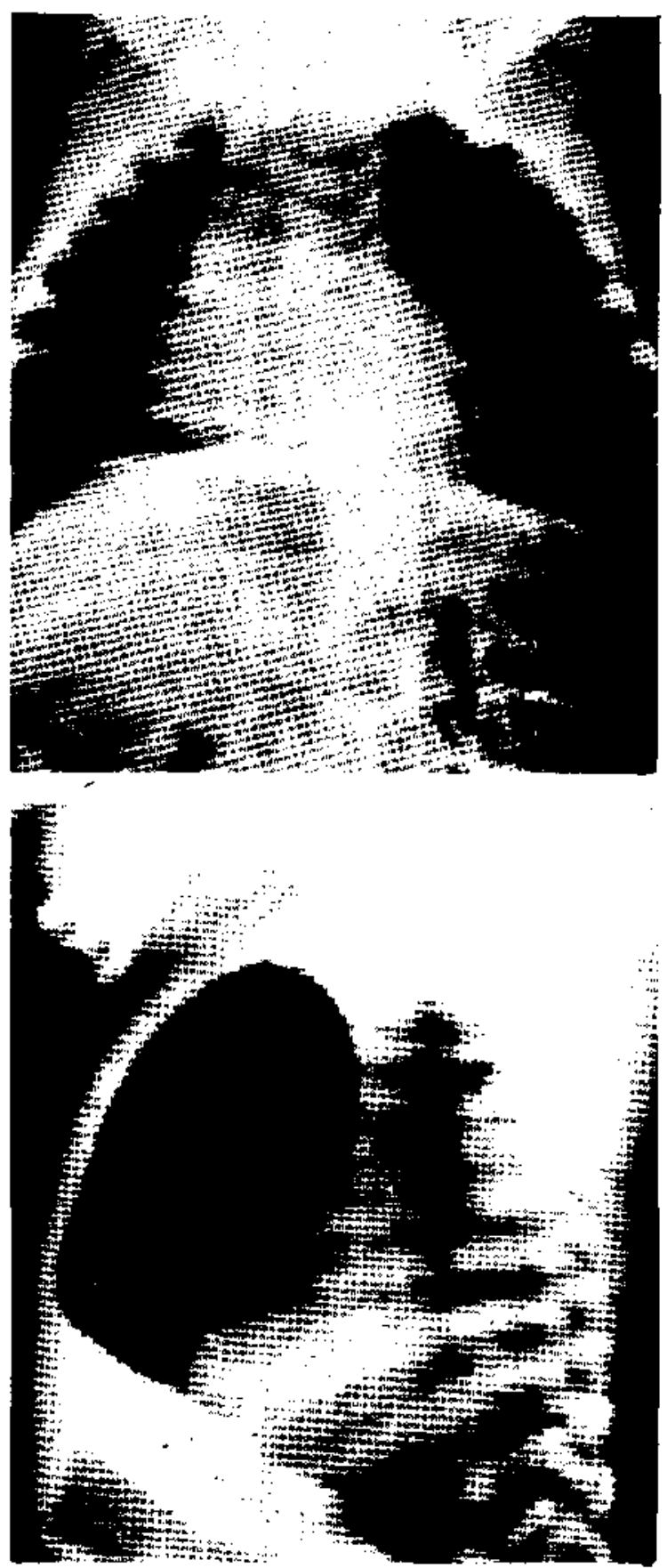
Se trata su cuedro coqueluchoideo con Eritromicina, KNT respiratorio y croupette, con lo cual evoluciona satisfactoriamente, luego de lo cual se interviene quirúrgicamente, comprobándose hiperinguflación pulmonar correspondiente a lóbulo superior y línguja izquierdo. Se practica lobectomia superior izquierda con buena evolución postoperatoria.

La biopsia concluye: presencia de alvéolos sobredistendidos, los que coexisten junto a proceso de atelectasia y hemorragia pulmonares, bronconeumonía focal y bronquiectasias. El control 2 años 2 meses postoperatorio muestra buena reexpansión del resto del pulmón izquierdo. Mediastino se encuentra en posición central, adherencias pleurales, egpecialmente mediastinales.

\section{CASO 3:}

Lactante de 2 1/2 meses de edad, eutrófico que ingresa por cuadro de dificultad respiratoria de instalación progresiva en el curso de 7 días.

Ex. F.: Polipnea, cianosis, retraceción subcostal, tórax enfitematoso.

Rgx de Tórax: Marcada hiperinsuflación del lóbulo pulmonar superior derecho que comprime el resto del pulmón hacia caudal y que atraviesa el mediastino y lo desplaza hacia la izquierda y atrás. Corazón iguadmente desplazado hacia posterjor y rotado. Ambos diafragmas bajos aplanados, especialmente el derecho. Sombras de condensación retrocardiaca izquierda. Enfisema pulmonar en el seno costodiafragmático izquierdo. Aumento del diámetro anteroposterior de la cavidad torácica y abombamiento de los espacios intercostales derechos.

Broncografía derecha: Bronquios del lóbulo medio y del lóbulo inferior aglutinados, desplazados hacia caudal del calibre normal. Bronquio principal del lóbulo euperior de calibre disminuido, desviado hacis caudal y contrastado sólo hasta $2 \mathrm{~cm}$. a partir de su fuente, donde la columna del medio de contraste termina abruptamente.

Se practica lobectomía auperior derecha, con buena reexpansión del L.M.D. y L.I.D.

La biopsia cornpatible con E.L.C.

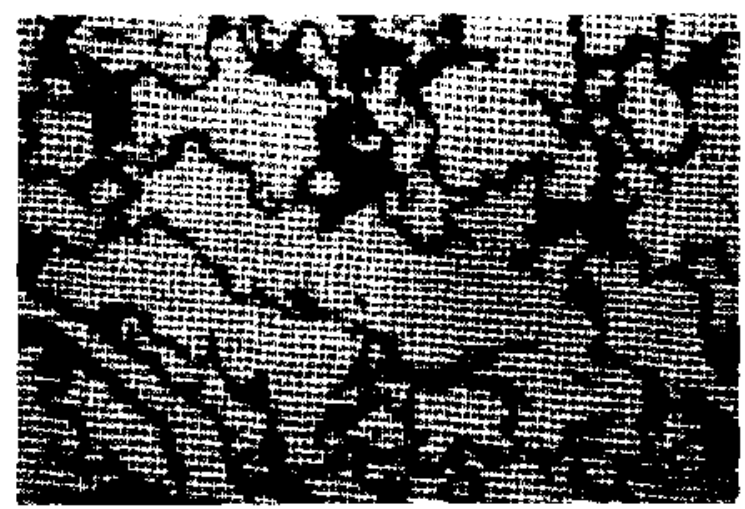

Evoluciona y es duda de alta en forma satisfactoria.

El control 16 meses después de la operación muestra completa reexpansión de los lóbulos medio e inferior y el mediastino en posición normal.

\section{DISCUSION}

La edad de inicio de la sintomatologia es variable, pero en general un alto número de casos se agrupa en el periodo neonatal o antes de los seis meses de vida. En concordancia con la bibliografía revisada, los casos distribuidos por sexo correspondieron a la relación 2:1 a favor del sexo masculino. (Gráfico 1.)

GAAFICO 1

,EDAD DE INICIO DE SINTOMATOLOGIA

EN 3 CASOS E.L.C.

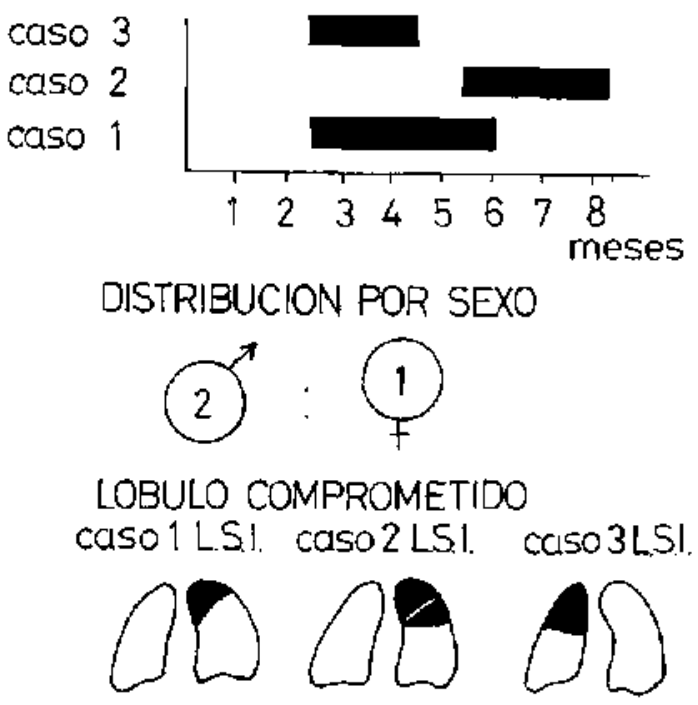

En general se ha reportado que cualquier lóbulo puede ser afectado, pero el lóbulo más frecuentemente comprometido corresponde al L.S.I. seguido por el L.M.D. y en tercer lugar el L.S.D.

En nuestra experiencia, dos casos se localizaron en L.S.I. y uno en L.S.D.

Son muy escasos los cuadros descritos que comprometen más de un lóbulo, o lóbulos inferiores.

La severidad de los sintomas del E.L.C. es variable, algunos niños presentan un cuadro de dificultad respiratoria inmediatamente después del parto, que requiere de solución quirúrgica inmediata, a diferencia de otros pacientes, como nuestros casos, que presentan síntomas en forma posterior y de grado menor, por lo que retarda el diagnóstíco. (Gráfico 2.) 


\section{GRAFICO 2}

\section{SINTOMATOLOGIA Y SIGNOLOGIA EN 3 CASOS E. L.C.}

\section{Disnea \\ Cianosis \\ Abombamiento \\ de Hemitorax \\ Hipersonoridad \\ a la Percusión \\ Signología Con - densación Asociada \\ Tonos Cardíacos Apagados}

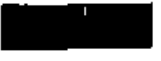

Los signos que se pesquisaron en nuestros casos fueron los de un sindrome de dificultad respiratoria caracterizado por disnea, taquipnea y cianosis; acompañado de abombamiento de hemitórax y signolugía de condensación asociada en dos casos; tonos cardiacos apagados en un caso.

En cierto número de casos es posible pesquisar hipersonoridad a la percusión y disminución o aboljción del murmullo vesjcular.

El cuadro radiológico del E.L.C. se caracteriza por una hiperclaridad habitualmente redondeada. que permite ver una estructura trabecular homogénea. Este fino sombreado, propio del tejido pulmonar, permite diferenciar al E.L.C. del pneumotórax. del quiste gigante, de la hernia diafragmática y de la enfermedad quística localizada del pulmón.

En el hemitórax afectado puede encuntrarse ensanchamiento de los espacios intercostales y el diafragma aparece aplanado. El mediastino se encuentra muy desviado hacia el lado sano y generalmente rechazado hacia atrás por el pulmón herniado, lo que se traduce en Rgx lateral en un aclaramiento retroesternal.

El otro pulmón aparece poco aireado, con algún lóbulo comprimido o atelactasiado.

La broncoscopía y broncografía son en general innecesarias para el diagnóstico, pero en oportunidades pueden presentar un complemento valioso.

En un número importante de casos de E.L.C. no se ha podido determinar una causa etiológica precisa (50\%).

Se sabe que el E.L.C. se encuèntra determinado por diversas causas, entre las cuales la más im- portante parece ser la "Displasia cartilaginosa bronquial", causa que tiende a ser descrita cada vez en forma más frecuente y que se manifiesta por un desarrollo deficiente del cartílago bronquial y por mucosas endobronquiales de tipo papilar.

La obstrucción intrínseca ha sido descrita frente a la presencia de pliegues de mucosa, determinados por una proliferación anormal de dicha mucosa endobronquial, que puede jugar un papel valvular en estos cuadros; también Munell y cols. han planteado lo que eilos denominan "löbulo polialveolar" como causa del E.L.C. En esta condición los alvéolos en su forma individual no son sobredistendidos, pero su número absoluto aumentado por acino pulmonar determina distensión aumentada de todo el lóbulo afectado.

La obstrucción extrínseca determinada por la compresión de un bronquio es posible frente a una anormalidad anatómica vascular, o a la compresión por nódulos hiliares hipertrofiados. Contró y cols. han descrito E.L.C. del lóbulo superior izquierdo secundario, a la dilatación aneurismática de la arteria pulmonar; Hendren y McKee del Jóbulo medio derecho por compresión del bronquio medio por la arteria pulmonar.

Se han descrito varios casos secundarios a una arteria pulmonar izquierda aberrante.

Landing y Wells describieron las alteraciones que podia prezentar el bronquio lobar. La más común es aquella en que el bronquia del lóbulo derecho proviene directamente de la tráquea. En ocasiones es posible observar la bifurcación en sólo dos bronquios del bronquio principal superior derecho.

Canty tecientemente publica un caso de E.L.C. determinado por una bifureación del bronquio lobar superior derecho a través de la arteria pulmonar.

Uno de nuestros casos presentó un bronquio supernumerario a lóbulo superior izquierdo que se origina en bronquiofuente cuya bistologia resultí normal.

La anormalidad difusa bronquial se ha presentado acompanada de un grado importante de engrosamiento de las paredes del bronquio periférico.

Se han descrito algunas malformaciones asociadas al E.L.C. tales como cardiopatías congénitas, malformaciones yasculares, hernias diafragmáticas, pectum excavatum, etc., que no pudimos observar en ninguno de nuestros casos.

Parece existir consenso hoy en día en la necesidad de la extirpación quirúrgica del lóbulo afectado en el E.L.C., justificado por el aumento progresivo de los síntomas, determinado por el incremento de 
la tensión intraalveolar y las neumonías que se asocian frecuentemente.

La mortalidad ha disminuido notoriamente con el tratamiento quirúrgico oportuno.

En nuestros casos no hemos tenido secuelas respiratorias posteriores a la lobectomía.

\section{RESUMEN}

Se presentan tres casos de Enfisema Lobar Congénito, controlados en el Hospital Exequiel González Cortés, en el período 1973-1978.

Todos correspondieron a lactantes menores de seis meses, con un periodo asintomático entre el parto y el inicio de la sintomatología, que consistió fundamentalmente en un síndrome de dificultad respiratoria de gravedad progresiva.

El cuadro radiológico consistió en una hipertransparencia con una estructura trabecular homogénea que correspondía a parénquima pulmonar.

Los tres pacientes fueron intervenidos, con buena evolución postoperatoria.
Se revisan las etiologías planteadas para el E.L.C. y las malformaciones asociadas descritas.

\section{REFERENCIAS}

I Leape, L. L., and Lungino, L. A. Intantile lobar emphisema. Pediarric's 34: 246-255, 1964.

2 Lincolu, j. R.C., etal. Gungenital Jobar emplusema. Avo. Surz. 173: 55-62. 1971.

3 Murray. G. F. Cingenital lubar emphisena. Sury. Gyn. Obstet. 124: $611-625.1567$.

4 Zatzkin, H. R, et al. Cingenilal hypertrophic lobar emphisema. Surgery 52: 505-512, \$ep. 62.

5 Maney, F. M. Jr., and Sabiston, D. C.Jr. The rule of pulnomonary scanning in the diagnosis of tongenital Jubar emphisena. Am. Surgeon 36: 20-27, Jan. 70 .

- DeLeca, F. G., et ut. Congenital lobar emphiseraa ducumented by serjal ruentusenograns. J. Ped. 82: 859-862, J973.

7 Kuffer, F., et et. Enphiseme lubaire congénital du mourrison. Helv. Paed. Aria 20: 236-250, 195.

8 Floyd, F. W, et al. Entisema lobar eougenito corregido quiruirgieanente. Actual. Ped. 26: [(1)]-194, 1964.

9 Herzoti, F., et al. El enfisemu lobar giganle del laclante. Actual. l'ed. 36; 167-169. 1\%69.

10 Bustais. W. L., et al. Lobur emplusema. iystic adenumajoid inalformation, pulmonary sequesiration, and bronchuyesic cyst in infancy and childbuad: a clinjeal greup.

Il cyutes. S. surgical respiratury emergencies in the newburn. J. Ped. Surg. 7: 558-559, 73 (Abstracts).

I2 Palucios, G. Entisema lobar cungénitu. Bol. Méd. Hospital Lifant. Mej. 36: 445-452, 79. 